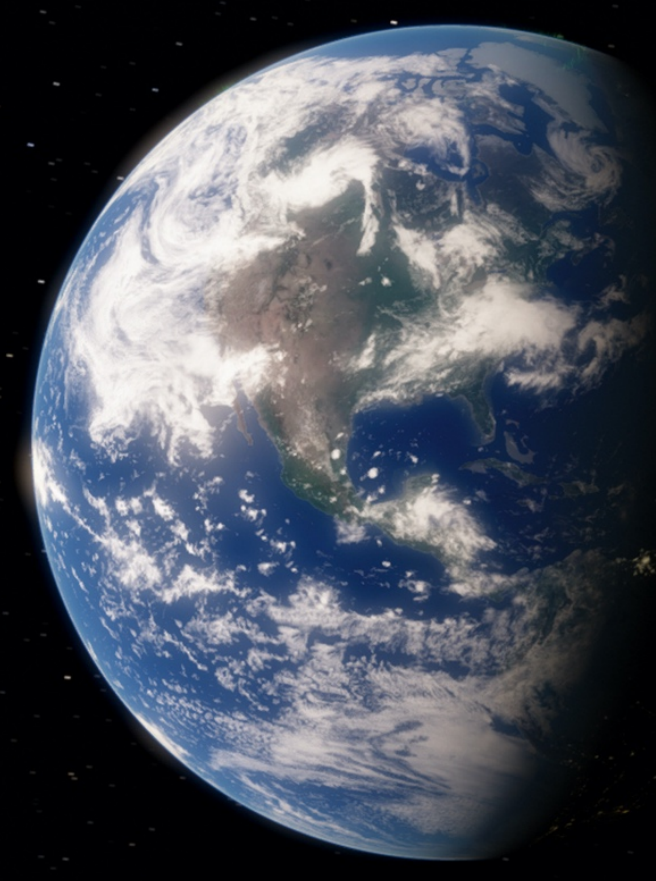

Original Research

\title{
Training Global Surgery Advocates: Strengthening the Global Surgery Voice
}

Xiya $\mathrm{Ma}^{1}$, Dominique Vervoot ${ }^{2,3}$, Dan Poenaru, MD4

MJM 2020 18(3)

\section{Abstract}

Objective: To strengthen knowledge of international medical trainees on global surgery and advocacy and help develop future generations of global surgeons, anaesthesiologists, and obstetricians.

Design: Training Global Surgery Advocates (TGSA), a standardized three-day advocacy workshop developed by the International Student Surgical Network (InciSioN), was built on traditional didactic lectures, role-play exercises, small working group activities, and advocacy and diplomacy training. Assessment was completed using a 5-point Likert scale for 18 components regarding the perceived familiarity, knowledge, and motivation for global surgery.

Setting: Training was provided in the context of the Pre-General Assembly of the International Federation of Medical Students Associations (IFMSA) at Université Laval, in Québec City, Canada.

\footnotetext{
${ }^{1}$ Faculty of Medicine, University of Montreal, Montreal, Quebec, Canada

2 Johns Hopkins Bloomberg School of Public Health, Johns Hopkins University, Baltimore, Maryland, United States of America

${ }^{3}$ Johns Hopkins Carey Business School, Johns Hopkins University, Baltimore, Maryland, United States of America

${ }^{4}$ Montreal Children's Hospital, McGill University Health Centre, Montreal, Quebec, Canada

Corresponding Author: Xiya Ma, email xiyama.xm@gmail.com
} 
Participants: Fourteen medical students from seven high-income countries and seven low- and middleincome countries participated in the workshop, from a group of twenty-five chosen applicants selected from a pool of 52 applicants.

Results: An average increase of 1.73 points across all 18 workshop components was observed among participants. After the workshop, all participants agreed or strongly agreed (4.64 average) that they were motivated to train other medical students in their respective countries to become global surgery advocates.

Conclusion: TGSA improved participants' knowledge and advocacy skills underlying global surgery. A mixed didactic and hands-on workshop appears to be feasible, enjoyable for participants, and effective in improving medical students' involvement in the emerging field of global surgery.

\section{Introduction}

In 2015, the Lancet Commission on Global Surgery reported that five billion people, or two-thirds of the world's population, lack timely access to safe and affordable surgical, obstetric, and anesthesia care when needed, causing over 17 million preventable deaths every year and responsible for one-third of the global burden of disease (1). Despite the proven costeffectiveness of surgical services in low-resource settings, the perceived luxury and difficulties of scaling up surgical care remain widespread (2).

Interest in global surgery-improving access to safe, timely, and affordable surgical care in low- and middleincome countries--has grown exponentially since the publication of the Lancet Commission on Global Surgery, especially amongst medical trainees. Nevertheless, availability or access to structured global surgery education in undergraduate or medical curricula is limited (3). Involvement of medical students and residents, however, has been successful in advancing the field of global surgery. InciSioN, the International Student Surgical Network, is an international non-profit organization run by, and for, medical and public health students, residents, and young physicians passionate to work in and advocate for global surgery (4). Formally established in 2016, the network has grown to over 5,000 members in over 80 countries and 39 fully established National Working Groups in countries across all world regions. These provide members with opportunities to become involved with research through peer-support mechanisms, virtual internships to learn from and work with global surgery experts, distance expert and peer mentorships, capacity building and soft skill development, as well as societal partnerships. Accordingly, InciSioN provides a platform to contribute to the development of future generations of global surgeons, anaesthesiologists, and obstetricians around the world.

As future physicians in the 21st century, medical trainees are called to develop not only clinical knowledge and expertise, but also interpersonal skills in order to meet the needs of a diverse population of patients. In 1990, the Royal College of Physicians and Surgeons of Canada developed the CanMEDS concept, which highlights the importance of the medical professional to develop various "soft skills" including communication, leadership, and most notably, health advocacy (5). The need for these skills is more pronounced in the global health and global surgery field, where social, economic, and cultural differences represent challenges on both the receiver and the provider's end of the care continuum.

To strengthen, unify, and escalate InciSioN's impact around the world, we have created Training Global Surgery Advocates (TGSA), a standardized three-day advocacy workshop. TGSA aims to equip participants with the essential knowledge of global surgery, universal health coverage, and health systems, as well as the advocacy and diplomacy skills to integrate this into meaningful communications and campaigns. We report results of the pilot program of this workshop, partaken by an international group of medical students convened in Quebec City, Canada.

\section{Methods \\ Course Design}

A three-day workshop was designed by InciSioN using feedback from InciSioN's core International Team and Board of Trustees on the proposed structure and content. The 27-hour workshop was built on traditional didactic lectures, role-play exercises, and small working group activities, as well as advocacy and diplomacy training in order to provide participants with the necessary knowledge and skills to effectively advocate for global surgery. One-third of the workshop curriculum was conducted through lectures serving as an introduction to global surgery, universal health coverage, health systems, and their constituents. The knowledge that participants gained was consequently integrated in active learning through advocacy training and peer-to-peer small working group activities. Participants were asked to complete both a baseline global surgery "elevator pitch" on the first day of the workshop, as well as a prepared "elevator pitch" at the end of the third day to personally assess progress and 
receive feedback from workshop facilitators and peers. The elevator pitches served as advocacy and persuasion practice to enable participants to more succinctly and efficiently deliver their messages to their intended audience.

\section{Participants}

A description and in-depth outline of the workshop was made available to medical students from the International Federation of Medical Student Associations (IFMSA) member organizations, who applied through a common form. Potential participants were asked to detail their motivation for participating in the TGSA, and to answer questions pertaining to global surgery. The quality of their answers, as well as their regional, country income group, and gender, were taken into account when selecting participants in order to ensure diversity and representation. From a pool of 52 applicants, 25 participants were selected in the initial round of evaluation, of which 14 medical students were able to attend from 14 different countries (7 highincome and 7 low- and middle-income countries, including Haiti, the Netherlands, Qatar, Ecuador, Turkey, Taiwan, Jordan, Denmark, Norway, Japan, Romania, Australia, India, and China). The remaining students were unable to attend due to visa issues (9 students) or personal reasons (2 students).

\section{Participant Assessment}

A questionnaire assessing familiarity with workshop topics and objectives was filled by participants before the beginning of the workshop and immediately afterwards (Appendix 1). Participants were asked to evaluate their personal motivation for future involvement within the field of global surgery. Questionnaires were scored using a 5-point Likert scale (strongly disagree, disagree, neutral, agree, strongly agree) for the 18 questions. The results from the pre- and post-workshop surveys were compared to determine changes for each component.

\section{Course Evaluation}

The workshop was evaluated by participants with comments at the end of each day. Each participant was required to give a minimum of one positive and one constructive comment on their satisfaction on the day's events. All comments were compiled and summarized according to which aspect of the workshop they addressed.

\section{Results and Discussion Course Selection}

During the three days, participants were exposed to a variety of topics designed to build a comprehensive foundation of global surgery and related topics, and given the opportunity to integrate these into practical interventions through public speaking, pitching, and campaigning (Table 1).

\section{Participants' Assessment}

Participants' self-assessment of their level of understanding of global surgery revealed various levels of experience in the field (Table 2). Statements pertaining to knowledge of global surgery and advocacy (statements 1 to 15) before the workshop range from virtually no experience (1 point, or strongly disagree) to extensive understanding of global surgery (5 points, or strongly agree), though the latter was limited to one participant. After the workshop, selfassessment on the same topics showed a notable increase in scores. In terms of evaluating knowledge (questions 1-15), overall, our cohort reported values of 2.28 points before the workshop and 4.28 points after the workshop, an increase of 2 points. Statements pertaining to motivation for becoming involved in global surgery (statements 16 to 18) showed high levels of motivation before the workshop (4.14 to 4.43 points), which were slightly increased after the workshop (0.14 to 0.5 point increase).

\section{Course Evaluation}

Feedback received through anonymous submissions from participants are summarized in Table 3. Overall, positive comments were more numerous than negative ones, as some participants gave no negative comments on some days. Many participants reemphasized their satisfaction with the content and structure of TGSA, which allowed them to translate acquired theory into practical applications, where they were able to receive feedback and improve over the course of the workshop.

\section{Discussion}

The Training Global Surgery Advocates workshop was a first of its kind, with the aim of introducing medical students to the basics of global surgery and equipping them with advocacy and diplomacy skills for future involvement in the field. This pilot study shows that a global surgery advocacy workshop, built on integration of theory into practice facilitated through discussion, reflections, and advocacy exercises, has a positive effect on participants' confidence regarding the topics of global surgery and universal health coverage.

Participant assessment shows that, on average, participants have increased their understanding across 
all topics that were presented within the TGSA and assessed via questionnaires. Our results on the assessment of knowledge of global surgery and advocacy, scored during pre- and post- workshop assessments, suggest that participants were generally not introduced to these topics within their respective medical curriculum, although many originated from countries where issues presented throughout the workshop were most prevalent. Participants informally commented on realizing the applicability of global surgery in their respective countries through discussion of challenges present in their medical systems regarding surgical care. Only modest improvements in self assessment responses for motivation in future involvement (questions 16-18) can be further explained by the high pre-workshop motivation of the majority of participants (average of 4.43, 4.29, and 4.14 preworkshop scores for local involvement in global surgery, international involvement in global surgery, and becoming a trainer for TGSA, respectively). Furthermore, an average score increase for all three of the motivation-related questions implies that overall, this workshop had a positive impact and encouraged future involvement in global surgery. Although the pilot study involved medical students, there is value to expanding and adapting future events to other audiences, including but not limited to residents, faculty, and other health professionals whose clinical duties pertain to surgery, anaesthesia, and obstetrics.

Short courses in medical education commonly report the use of numerical scales in questionnaires as a means to obtain feedback and evaluate participants learning (6-7). Notably, self-assessment is widely accepted as a simple but effective measure to assess participants' understanding of a topic. Our assessment was based on the use of the Likert scale as a simple quantitative tool to evaluate improvement in participants' perception of their understanding of global surgery before and after the workshop. The Likert scale is a simple instrument that detects selfreported changes and has been widely used and validated in clinical settings (9-11). Additionally, in our study, open-ended qualitative feedback was incorporated to optimize future workshops.

\section{Limitations}

Within this study, one limitation was the number of participants who were unable to attend the workshop due to visa issues. Fourteen medical students participated in the TGSA, although the initial number of accepted participants was 25 , which creates a sampling bias. This problem is concerning as creates limitations in representation from some regions of the world, particularly for participants from sub-Saharan Africa and is regrettably a widespread phenomenon for international conferences and workshops (12). A potential solution to this problem would be to establish an e-TGSA, or online version of the training, which could be conducted as a series of webinars. This method would retain the interactive and participative aspect of the TGSA, whilst making the content accessible to anyone. An online workshop is also attractive due to its modest cost for both facilitators and participants, as it removes the need to physically be present at a specific location. However, online workshops lack a face-to-face component, which would thereby limit the ability of participants to foster relationships with future colleagues during and between sessions, an aspect which was repeatedly highlighted as an important strength of the workshop. Additionally, the quality of the training may vary depending on the quality of the internet connection, and it would be more challenging to determine whether participants are actively engaged.

Another challenge faced by the TGSA workshop is the physical and human resources needed to hold it. This pilot run was designed to allow in-person interactions and feedback, which have limitations as to who and when it can be delivered. A possible future direction for TGSA would be to re-purpose it as a template workshop, which would allow the creation of similar workshops that are catered to the needs of the attendees. Alternatively, an online module could be developed based on the workshops, which would increase accessibility but limit the in-person interactions of TGSA.

Finally, the self-assessment nature of our evaluation method is prone to cognitive bias and the DunningKruger effect, whereas participants could be overestimating their knowledge and skills when answering the survey questions (13). This could explain why, on an individual level, some participants have not indicated an improvement for some topics, as their experience with the training may have clarified how their knowledge and competencies compare to other participants.

\section{Conclusions}

Global surgery is a young and rapidly developing field, hence naturally prone to gaps in understanding feasibility, cost-effectiveness, and the urgent need of scaling up surgical systems. InciSioN's standardized three-day advocacy workshop, Training Global Surgery Advocates, aimed to equip participants with the needed knowledge, as well as advocacy and 
diplomacy skills to advocate for global surgery, adaptable in any situation and to any target audience. This pilot study of TGSA suggests that this type of workshop is feasible, enjoyable for participants, and efficient in improving medical students' knowledge and involvement in the emerging field of global surgery.

\section{Appendix A: Pre- and Post-Workshop Survery}

Pre- and post-workshop survey statements (rated on a scale of 1 to 5,1 being "strongly disagree" to 5 being "strongly agree")

1. I have a good understanding of global surgery.

2. I have a good understanding on how nongovernmental organizations (NGOs) work for global surgery.

3. I have a good understanding on how academic centres and universities work for global surgery.

4. I have a good understanding on how governments work for global surgery.

5. I have a good understanding on the different ways a student can get involved in global surgery.

6. On a scale of 1-5, how well is your understanding of...
a. The Lancet Commission on Global Surgery
b. UHC2030
c. Financial Risk Protection
d. The Bellwether Procedures
e. InciSioN - International Student Surgical Network
f. National Surgical, Obstetric, and Anaesthesia Plans (NSOAPs)
g. Catastrophic Expenditure
h. Health Systems Financing
i. Social Determinants of Health
j. Advocacy

7. I am motivated to get involved in global surgery locally in my country.

8. I am motivated to get involved in global surgery on the international level.

9. I want to train other students to become global surgery advocates.

\section{References}

1. Meara JG, Leather AJM, Hagander L, Alkire BC, Alonso N, Ameh EA, et al. Global Surgery 2030:

Evidence and solutions for achieving health, welfare, and economic development. Lancet.
2015;386(9993):569-624. doi:

10.1016/j.ijoa.2015.09.006.

2. Grimes CE, Henry JA, Maraka J, Mkandawire NC, Cotton M. Cost-effectiveness of surgery in low- and middle-income countries: A systematic review. World J Surg. 2014;38(1):252-63. doi: 10.1007/s00268-0132243-y.

3. Scott EM, Fallah PN, Blitzer DN, NeMoyer RE, Sifri Z, Hanna JS, et al. Next Generation of Global Surgeons: Aligning Interest with Early Access to Global Surgery Education. J Surg Res. 2019 Apr;240:219-26. doi: 10.1016/j.jss.2019.03.009.

4. Vervoort D, Bentounsi Z. InciSioN: Developing the Future Generation of Global Surgeons. J Surg Educ. 2019 Jul-Aug;76(4):1030-1033. doi: 10.1016/j.jsurg.2019.02.008.

5. Doiron RC. Health advocacy in a competency-based curriculum: The emerging role of global surgery. Can Urol Assoc J. 2016 Mar-Apr;10(3-4):82. doi: 10.5489/cuaj.3771.

6. Schoeb DS, Brennecke E, Andert A, Grommes J, von Trotha KT, Prescher A, Neumann UP, Binnebösel M. Assessment of a course of realistic surgical training during medical education as a tool for pre-residential surgical training. BMC Med Educ. 2016 Feb 3;16:45.

7. Rempell JS, Saldana F, DiSalvo D, Kumar N, Stone MB, Chan W, Luz J, Noble VE, Liteplo A, Kimberly H, Kohler MJ. Pilot Point-of-Care Ultrasound Curriculum at Harvard Medical School: Early Experience. West J Emerg Med. 2016 Nov;17(6):734-740. Epub 2016 Sep 12.

8. Ross JA. The Reliability, Validity, and Utility of SelfAssessment. Practical Assessment, Research \& Evaluation 2006, 11(10). Available online: http://pareonline.net/getvn.asp?v=11\&n=10.

9. Krzych ŁJ, Lach M, Joniec M, Cisowski M, Bochenek A. The Likert scale is a powerful tool for quality of life assessment among patients after minimally invasive coronary surgery. Kardiochir Torakochirurgia Pol. 2018 Jun;15(2):130-134. doi: 10.5114/kitp.2018.76480.

10. Koksel Y, Gencturk M, Spano A, Reynolds M, Roshan S, Caycı Z. Utility of Likert scale (Deauville criteria) in assessment of Chemoradiotherapy response of primary oropharyngeal squamous cell Cancer site. Clin Imaging. 2019 May - Jun;55:89-94. doi: 10.1016/j.clinimag.2019.01.007.

11. van Laerhoven $\mathrm{H}$, van der Zaag-Loonen HJ, Derkx BH. A comparison of Likert scale and visual analogue scales as response options in children's questionnaires. Acta Paediatr. 2004 Jun;93(6):830-5.

12. Nshemereirwe $C$. Tear down visa barriers that block scholarship. Nature. 2018 Nov;563(7729):7. doi: 10.1038/d41586-018-07179-2.

13. Kruger J, Dunning D. Unskilled and unaware of it: how difficulties in recognizing one's own incompetence lead to inflated self-assessments. J Pers Soc Psychol. 1999 Dec;77(6):1121-34.

\section{Tables}


Table 1: Outline of the TGSA workshop

Day 1

Ice Breaker

Introduction to Universal Health Coverage

Introduction to Health Systems

Introduction to Global Surgery

Baseline Elevator Pitch

The Three Delays Framework

Human Resources for Surgery, Anaesthesia, and

Obstetrics

Table 2: Participants' self-assessment before and after attending the workshop

\section{Statement}

1. I have a good understanding of global surgery.

2. I have a good understanding on how non-governmental $2.36(0.93)$ organizations (NGOs) work for global surgery.

3. I have a good understanding on how academic centres $2.57(0.94)$ and universities work for global surgery.

4. I have a good understanding on how governments work $2.43(0.94)$ for global surgery.

5. I have a good understanding on the different ways a $\quad 2.69(0.63)$ student can get involved in global surgery.

6. On a scale of $1-5$, how well is your understanding of... $1.92(0.95)$

[The Lancet Commission on Global Surgery]

7. On a scale of $1-5$, how well is your understanding of... 2.54 (1.05)

[UHC2030]

8. On a scale of $1-5$, how well is your understanding of... $1.85(0.69)$

[Financial Risk Protection]

9. On a scale of $1-5$, how well is your understanding of... $\quad 1.46(0.78)$

[The Bellwether Procedures]

10. On a scale of $1-5$, how well is your understanding of... $2.08(0.95)$

[InciSioN - International Student Surgical Network]

11. On a scale of 1-5, how well is your understanding of... 2.08 (1.04)

[National Surgical Plans (NSOAPs)]

12. On a scale of $1-5$, how well is your understanding of... $1.62(0.65)$

[Catastrophic Expenditure]

13. On a scale of $1-5$, how well is your understanding of... $2.31(0.75)$

[Health Systems Financing]

14. On a scale of $1-5$, how well is your understanding of... 2.5 (1.02)

[Social Determinants of Health]

15. On a scale of $1-5$, how well is your understanding of... 3.07 (1.14)

[Advocacy]

16. I am motivated to get involved in global surgery locally $4.43(0.76)$

in my country.

17.I am motivated to get involved in global surgery on the $4.43(0.65)$ international level.

18. I want to train other students to become global surgery $4.14(0.53)$

advocates.
Day 2

Day 3

Rural Health and Rural Surgery

Academic Global Surgery

Global Surgery in Medical

Education

Health Systems Sustainability

Global Surgery Campaign

Creation

Final Elevator Pitch

Debrief and Closing
Post-assessment average Average change (Standard deviation)

$4.79(0.43)$

$4.21(0.70)$

$+2.14(0.95)$

$+1.86(1.03)$

$+1.86(0.86)$

$4.43(0.51)$

$+1.64(0.84)$

$4.07(0.62)$

$4.92(0.28)$

$+2.21(0.70)$

$3.69(0.63)$

$+1.71(0.82)$

$4.08(0.76)$

$+1.64(1.01)$

$3.85(0.80)$

$+2(0.78)$

$4.54(0.66)$

$+3(1.11)$

$4.46(0.66)$

$+2.43(1.16)$

$4.31(0.63)$

$+2.29(1.20)$

$4.31(0.75)$

$+2.64(0.84)$

$3.69(0.95)$

$+1.5(1.29)$

$4.29(0.91)$

+1.79 (1.42)

$4.5(0.94)$

$+1.43(1.40)$

$4.86(0.36)$

$+0.43(0.65)$

$4.57(0.65)$

$+0.14(0.77)$

$4.64(0.50)$

$+0.5(0.52)$ 
Table 3: Course evaluation by participants

\begin{tabular}{|c|c|c|}
\hline Aspect evaluated & Positive feedback & Constructive feedback \\
\hline $\begin{array}{l}\text { Content of the } \\
\text { workshop }\end{array}$ & $\begin{array}{l}\text { 1. Variety of topics } \\
\text { 2. Pertinent and enjoyable exercises, especially for } \\
\text { advocacy and soft skills (e.g., elevator pitch) }\end{array}$ & $\begin{array}{l}\text { 1. Depth of some topics to re-assess depending on } \\
\text { participants' level of knowledge }\end{array}$ \\
\hline $\begin{array}{l}\text { Structure of the } \\
\text { workshop }\end{array}$ & $\begin{array}{l}\text { 1. Opportunities to put theory into practice } \\
\text { 2. Balance between theoretical presentations and } \\
\text { practical workshops }\end{array}$ & $\begin{array}{l}\text { 1. Room set-up to be improved } \\
\text { 2. Time management (longer duration of activities, order of } \\
\text { the workshops, punctuality of participants) }\end{array}$ \\
\hline Social interaction & $\begin{array}{l}\text { 1. Ice breaker activity } \\
\text { 2. Diversity of participants' background }\end{array}$ & 1. Lack of group activities beyond the workshop hours \\
\hline
\end{tabular}

